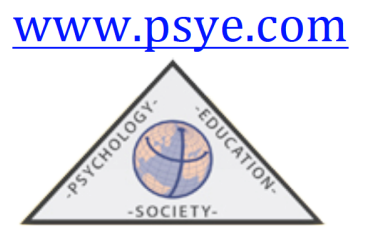

\title{
Diseño y validación de la Escala de Tiempo Compartido en la Pareja (TCP)
}

\author{
Antonio URBANO-CONTRERAS, M M $^{\text {a }}$ Teresa IGLESIAS-GARCÍA \\ y Raquel Amaya MARTÍNEZ-GONZÁLEZ
}

Universidad de Oviedo

(Recibido, el 7 de Marzo de 2018; Aceptado el 29 de Enero de 2019)

\begin{abstract}
RESUMEN: Este estudio busca diseñar y validar la Escala de Tiempo Compartido en la Pareja (TCP) con el fin de ofrecer un instrumento útil y sencillo de aplicar. Han participado 620 personas que mantenían una relación de pareja (el $57.7 \%$ eran mujeres, el 51.6\% tenía más de 31 años, el $66.1 \%$ convivía con su pareja y el $57.9 \%$ no tenía hijos). La muestra total se dividió en dos grupos, con el objetivo de desarrollar con el primero un Análisis Factorial Exploratorio (AFE) y con la mitad restante un Análisis Factorial Confirmatorio (AFC), estrategia denominada validación cruzada. Los resultados ofrecen una escala con una buena fiabilidad $(\alpha=.80)$, que explica el $55 \%$ de la varianza y con una estructura bifactorial integrada por 7 ítems. Su brevedad y validez indican que este instrumento puede ser de utilidad para la investigación y la práctica profesional, especialmente en el campo de la prevención y detección de necesidades.
\end{abstract}

Palabras clave: Relación de pareja; Uso del tiempo; Validación de instrumentos; Análisis factorial

\section{Design and validation of the Couple Shared Time Scale (CST)}

\begin{abstract}
This study aims to design and validate the Couple Shared Time Scale (CST) in order to provide a useful measure in the field of Couple Relationship. 620 participants who were in a couple relationship took part in the study $(57.7 \%$ were women, $51.6 \%$ were aged 31 or older, $66.1 \%$ lived with their partner and $57.9 \%$ did not have children. The main sample was divided into two groups to perform an Exploratory Factor Analysis (EFA) with the first group, and a Confirmatory Factor Analysis (CFA) with the remaining half, strategy known as cross-validation. Results show a scale with a good reliability $(\alpha=.80)$, explaining the $55 \%$ of variance, with a bifactorial structure made up with 7 items. Its short length and validity indicate that this instrument can be useful for both research and professional practice, especially in the areas of needs prevention and detection.
\end{abstract}

Keywords: Couple relationship; Use of time; Instrument validation; Factor analysis

Correspondencia: $\mathrm{M}^{\mathrm{a}}$ Teresa Iglesias-García. Facultad de Formación del Profesorado y Educación. Universidad de Oviedo. C/ Aniceto Sela, s/n, 33005, Oviedo, Principado de Asturias (España). E-mail: teresai@uniovi.es 


\section{Introducción}

La relación de pareja y la vida familiar constituyen una dimensión central en la vida de las personas que parece suscitar cada vez mayor atención e interés científico y social (Torres, 2004). Numerosas investigaciones han puesto de manifiesto la influencia que tiene la forma en que se vive una relación de pareja en aspectos tan fundamentales como la felicidad, la salud y la dinámica familiar, pues las consecuencias de su adecuado o inadecuado funcionamiento repercuten en cuestiones como la salud mental de las personas del entorno o la calidad de las relaciones entre padres e hijos (Capafóns y Sosa, 2015; Çetinkaya y Gençdoğan, 2014; Leggett, Roberts-Pittman, Byczek y Morse, 2012; Martínez-Álvarez, Fuertes-Martín, Orgaz-Baz, Vicario-Molina y González-Ortega, 2014).

Puesto que las relaciones de pareja están configuradas por diversas dimensiones interconectadas, es posible encontrar innumerables temas de estudio que abordan este tipo de relaciones humanas. A pesar de que la investigación e intervención en el ámbito de la pareja cada vez incorporan más enfoques y modelos preventivos a sus líneas de trabajo, se observa que algunos temas, como la satisfacción sexual, muestran cierta tendencia a ser analizados desde un prisma fundamentalmente clínico o no incluir a ciertos colectivos como las personas mayores (Faus-Bertomeu y Gómez-Redondo, 2017), lo que puede conllevar un sesgo importante y evidencia la necesidad de profundizar en ellos desde una perspectiva de funcionalidad y preventiva (Fuertes Martín, 2000; Urbano Contreras, 2018).

Partiendo de la idea de que las relaciones de pareja están determinadas por sus propias características y por el contexto cultural y social en el que se desarrollan, van a ser muy diversos los aspectos que influyan en las dinámicas de interacción, el ajuste y la calidad de estas relaciones (Wals, Romera y Viejo, 2015). Entre los elementos que configuran el día a día en pareja, pasar tiempo juntos y comunicarse aporta la posibilidad de compartir e intercambiar información sobre sus valores, experiencias, sentimientos y proyectos de vida (Padilla González, Armas Vargas, García Pérez y Aguiar González, 2012), por lo que la falta de oportunidades para poder llevar a cabo el acto comunicativo influirá, inevitablemente, en el bienestar personal y en el de la pareja.

De entre los diversos momentos evolutivos que puede experimentar una pareja, convertirse en padres parece ser uno de los que mayor impacto suele tener en las dinámicas de las parejas pues, por ejemplo, suele conllevar modificaciones en los patrones de uso del tiempo y en su distribución entre los miembros de la pareja (Domínguez Folgueras, 2012). Con la llegada de los hijos suelen verse modificados los hábitos personales y las rutinas propias de la relación de pareja, con una especial disminución del tiempo dedicado a las actividades de ocio y al tiempo compartido (Hidalgo y Menéndez, 2003; 2009), lo que puede estar asociado con el descenso de la satisfacción con la relación de pareja cuando se tiene hijos (Mora Torres, Gomez Cortés y Rivera Heredia, 2013; Urbano-Contreras, Martínez-González e IglesiasGarcía, 2018).

Este cambio inicial en la disponibilidad y el empleo del tiempo en pareja se extiende durante la primera infancia de los hijos, durante la cual se crean lazos de dependencia y la asunción de funciones ineludibles con respecto al hijo, lo que acapara gran parte del tiempo libre o de ocio con el que contaba la pareja (Ríos González, 2005). En este sentido, los resultados de otras investigaciones ponen de relieve que las madres son las que pasan más 
tiempo con los hijos, quedando patente la falta de igualdad real en la corresponsabilidad educativa entre los miembros de la pareja (García Román, 2013). Incluso estudios más recientes (Moreno-Colom, Ajenjo Cosp y Borràs Català, 2018) siguen reflejando la dedicación desigual que se da entre hombres y mujeres con respecto al trabajo doméstico y la segregación de tareas según su contenido, aunque al menos recogen cierta disminución de dichas diferencias. En función de los resultados que continúan señalando la falta de igualdad en el trabajo doméstico y en el cuidado de los hijos, sería conveniente llevar a cabo investigaciones que intenten comprobar si estas cuestiones guardan relación, por ejemplo, con un menor grado de satisfacción con la relación de pareja por parte de las mujeres (Urbano-Contreras, IglesiasGarcía y Martínez-González, 2019).

Además de la influencia directa en la satisfacción con la relación de pareja, disponer de tiempo y emplearlo adecuadamente está muy relacionado con las oportunidades de comunicarse y la calidad de la propia comunicación, para lo que es fundamental no considerar únicamente la posible falta de tiempo, sino también si aquel que se tiene disponible, sea mucho o poco, se emplea adecuadamente. Analizar los usos del tiempo en la pareja permite profundizar en el estudio de la comunicación y proponer acciones de mejora, lo que también puede contribuir al incremento de la calidad de otras dimensiones en la relación de pareja, como la satisfacción general (Ledermann, Bodenmann, Rudaz y Bradbury, 2010).

Por último, el uso adecuado del tiempo en las relaciones de pareja, también parece influir notablemente en otros aspectos como son los conflictos en la relación. Por ejemplo, el tiempo que se dedica al trabajo o el estrés que causan las propias actividades laborales pueden ser fuentes de conflicto que acaben afectando a otros ámbitos, como la convivencia, al verse claramente delimitadas las actividades compartidas por la pareja (Armenta-Hurtarte, SánchezAragón y Díaz-Loving, 2012). Al considerar esta cuestión, resulta de interés intentar predecir situaciones que puedan contribuir al incremento de conflictos $y$, por lo tanto, a la disminución de la satisfacción y el bienestar en la relación de pareja.

Ante los estudios que señalan los cambios en la disponibilidad y el uso del tiempo tras acontecimientos tan cruciales como la llegada de los hijos, o la influencia de esta dimensión en otras tan importantes como la satisfacción con la relación, la comunicación o los conflictos, es necesario contar con instrumentos válidos y fiables que contribuyan a medir y contrastar si el tiempo disponible y el empleo del mismo es diferente, por ejemplo, entre personas que son o no padres, entre hombres y mujeres o en función del tiempo de duración de la relación. De igual modo, la creación y validación de un instrumento centrado en la dimensión tiempo, también puede resultar de utilidad para analizar cómo correlaciona esta esfera con otras ya ampliamente estudiadas y con un largo recorrido en la construcción de instrumentos de evaluación de la relación de pareja. Por ello, este estudio busca diseñar y validar la Escala de Tiempo Compartido en la Pareja (TCP), con el fin de que resulte un instrumento útil y de fácil aplicación que pueda ser utilizado en el ámbito de la investigación y en el de la intervención con parejas.

\section{Método}

\section{Participantes}

Han participado de forma voluntaria y sin remuneración 620 personas, de las cuales el $57.7 \%$ eran mujeres y el $42.3 \%$ hombres que en el momento de responder mantenían una 
relación de pareja. En cuanto a su edad, el 48.4\% tenía entre 18 y 31 años y el $51.6 \% 32$ años o más. Respecto al nivel de estudios, el 53\% contaba con estudios universitarios, el $28.1 \%$ había cursado bachillerato o Formación Profesional, el 16.6\% Educación Secundaria y el 2.3\% no tenía estudios. En lo laboral, el 52.3\% trabajaba, el 23.4\% era estudiante, el 19.7 desempleado y el $4.6 \%$ jubilado.

Atendiendo a la situación como pareja, el $54.5 \%$ de los sujetos estaba soltero y el $45.5 \%$ casado o constituido como pareja de hecho. El tiempo de duración de la relación era de dos años o menos en el $19.4 \%$ de los casos, de entre 3 y 5 en el $20.6 \%$, de entre 6 y 10 en el $18.7 \%$, de entre 11 y 20 en el 17.4\% y el 23.9\% llevaba juntos más de 20 años (12.1 años de media, mediana de 8 años). El 57.9\% indicaba no tener hijos y el 42.1\% sí eran padres. Por último, el $66.1 \%$ convivía en ese momento con su pareja y el $46.9 \%$ no había tenido ninguna otra relación con anterioridad.

\section{Instrumento}

Para la elaboración del cuestionario se siguieron las fases indicadas en la literatura especializada sobre este tipo de técnicas (Gil Pascual, 2011). Inicialmente, se redactó un instrumento con 23 preguntas con respuesta en escala Likert de cuatro alternativas $(1=$ totalmente en desacuerdo a 4 = totalmente de acuerdo, evitando así la tendencia a un valor intermedio), las cuales fueron elaboradas por los autores basándose en cuestiones que la literatura científica indicaba como relevantes y vinculadas a la disponibilidad y el empleo del tiempo en las relaciones de pareja. Este cuestionario fue revisado en un primer momento por un grupo de expertos en métodos de investigación y, posteriormente, por otro grupo de expertos en intervención familiar y relaciones de pareja. Con las sugerencias recibidas se redactó una segunda versión que se aplicó, a modo de prueba, a un grupo de personas de características sociodemográficas diversas para comprobar si su cumplimentación resultaba fácil y clara. Como resultado se modificaron parte de los ítems en base a las sugerencias y comentarios aportados.

Con el fin de analizar la validez concurrente del instrumento diseñado, se incluyeron dos ítems o indicadores externos en la versión definitiva del cuestionario para valorar, por un lado, la satisfacción general con la relación de pareja (Estoy satisfecho con mi relación de pareja) y, por otro, la calidad del tiempo compartido por la pareja (Estoy satisfecho con la calidad del tiempo que compartimos mi pareja y yo). Estos dos ítems no pueden considerarse como parte de la escala final, ya que fueron seleccionados por considerarlos relevantes en la literatura revisada y para evitar solicitar a los participantes que cubrieran otros instrumentos, dada la falta de motivación que esto puede generar.

\section{Procedimiento}

Se accedió a la muestra mediante el método no probabilístico "Bola de nieve", iniciando el proceso de recogida de información con parejas conocidas que, además de cumplimentar el cuestionario, actuaron como enlace a otras parejas de su entorno y estas, a su vez, a otras. Para recoger la información se optó por dos procedimientos: en unos casos, se entregó de forma presencial a cada persona un cuestionario y un sobre, de tal forma que aunque los dos miembros cubrieran el cuestionario ninguno tuviera acceso a la información del otro; en otros 
casos, para llegar a más parejas, también se informatizó el cuestionario con la herramienta de Formularios de Google y se envió el enlace a personas disponibles para participar en el estudio, que pudieran a su vez hacer llegar el cuestionario online a sus contactos cercanos. Previamente a iniciar la recogida de información se establecieron dos áreas geográficas distanciadas de España (Asturias en el norte y Málaga en el Sur) con el fin de incrementar la diversidad de la muestra obtenida.

\section{Análisis de datos}

Previo al inicio de los análisis estadísticos, se revisó la base de datos para descartar valores perdidos y casos atípicos, que podrían sesgar los análisis posteriores; para ello se aplicó la prueba MCAR (Little, 1988). Posteriormente se comprobó el ajuste de los ítems a la distribución normal analizando la asimetría y curtosis en caso de que estos valores pudieran condicionar análisis multivariados posteriores; se estableció como criterio que la asimetría se situara por debajo del valor 2 y la curtosis por debajo del valor 7 (Curran, West y Finch, 1996) y se eliminaron aquellos ítems que no cumplieran dicho criterio. Se analizó también la multicolinealidad entre los ítems, calculando las correlaciones bivariadas inter-ítem para poder observar el grado y el sentido de las relaciones existentes entre los mismos; se consideraron válidas aquellas que no reflejaran un $\mathrm{r} \geq .90$ (Tabachnick y Fidell, 2001).

Para estudiar la estructura factorial o validez de constructo, se llevó a cabo un proceso de validación cruzada con análisis factorial exploratorio (AFE) y análisis factorial confirmatorio (AFC) dividiendo la muestra inicial en dos secciones equivalentes de 310 sujetos cada una, separando a los dos miembros de la pareja en los casos en que se contaba con la respuesta de ambos. La primera submuestra quedó compuesta por 262 hombres y 48 mujeres y la segunda submuestra por 310 mujeres. Con la primera submuestra se realizó un análisis factorial exploratorio (AFE) utilizando el método de extracción de máxima verosimilitud, ya que la literatura sugiere este método cuando se cumple el supuesto de normalidad (Fabrigar, MacCallum, Wegener y Strahan, 1999) y el método de rotación promax, ya que el método oblicuo resulta más efectivo en la identificación de una estructura simple (Finch, 2006); los supuestos de aplicación se verificaron con la medida de adecuación muestral de Kaiser-MeyerOlkim y el Test de esfericidad de Bartlett.

Con la segunda submuestra se realizó un análisis factorial confirmatorio (AFC) utilizando la estimación de máxima verosimilitud, evaluándose la bondad de ajuste del modelo propuesto mediante una combinación de índices de ajuste absolutos y relativos. Entre los absolutos, se utilizó el valor p, asociado con el estadístico chi cuadrado, que prueba el modelo nulo frente al modelo hipotetizado y el valor de la ratio entre $\chi^{2}$ y los grados de libertad (CMIN/DF), que es un heurístico que se utiliza para reducir la sensibilidad del $\chi 2$ al tamaño de la muestra. No obstante $\mathrm{y}$, dada la sensibilidad mostrada por la prueba de $\chi^{2}$ al tamaño muestral, se calcularon otros índices de ajuste absoluto, como el índice de bondad de ajuste (GFI) [The Goodness of Fit Index], la raíz cuadrática media de residuales (SRMR) [Standardized Root Mean Square Residual] y el error cuadrático medio de aproximación (RMSEA) [Root Mean Square Error of Approximation], así como los siguientes índices incrementales: índice de ajuste comparado (CFI) [Comparative Fit Index], el índice de ajuste normado (NFI) [The Goodness of Fit Index], el índice de ajuste incremental (IFI) [Incremental 
Fit Index] y el índice ajustado de bondad de ajuste (TLI) [Tucker Lewis Index]. Se consideró que para que existiera un buen ajuste, el valor CMIN/DF debía ser inferior a 2 (Tabachnik y Fidell, 2001); el valor de GFI estar por encima de .95 (Hoelter, 1983); los valores NFI, TLI, IFI y CFI debían superar el valor de .95 , aunque no deben considerarse como puntos de corte fijos (Markland, 2007); y que valores de RMSEA y SRMR inferiores o muy próximos a .06 y .08 se considerarían, respectivamente, como indicativos de un adecuado ajuste del modelo a los datos (Hair, Black, Babin, Anderson y Tatham, 2006).

La consistencia interna o fiabilidad del cuestionario se estableció mediante el coeficiente alfa de Cronbach, calculándose también la correlación ítem-total corregido y el valor alfa de Cronbach si el ítem era eliminado. Los valores de alfa mayores o iguales a .90 se consideraron excelentes, de .80 a .89 buenos, de .70 a .79 aceptables, de .60 a .69 cuestionables, de .50 a .59 pobres y los inferiores a .50 inaceptables (Cronbach y Shavelson, 2004).

Por último, se analizó la validez concurrente mediante la correlación de Pearson entre los factores resultantes y las variables externas seleccionadas previamente como variables criterio (Estoy satisfecho con mi relación de pareja y Estoy satisfecho con la calidad del tiempo que compartimos mi pareja y yo). Según la literatura revisada, se consideró que estos ítems podían utilizarse como indicadores o criterios externos de lo que el instrumento pretendía medir, esperando encontrar cierto grado de relación estadística entre las respuestas obtenidas en dichos ítems y los factores del cuestionario.

Para analizar los datos recogidos se utilizó el paquete estadístico SPSS 22.0, incluido el módulo AMOS 22.0.

\section{Resultados}

\section{Análisis factorial exploratorio}

El porcentaje de valores perdidos se encontraba entre $0.3 \%$ y $1.9 \%$ y los resultados obtenidos en la prueba MCAR fueron $\chi 2=754.796, \mathrm{DF}=682, \alpha=.027$, por lo que concluimos que los datos perdidos no son MCAR (Falta Completamente al Azar). Por ello, ha sido necesario aplicar la estimación EM (Expectation-Maximization), utilizando el módulo de Análisis del Valor Perdido del programa SPSS, ya que este procedimiento tiene claras ventajas en contextos aplicados (Van Ginkel y Van der Ark, 2005).

Todos los ítems cumplieron los supuestos de la curva normal, con valores por debajo de 2 en asimetría y por debajo de 7 en curtosis, con excepción del ítem "Tengo confianza en mi pareja" (asimetría: -2.236 y curtosis 5.519), por lo que ha sido excluido del análisis, quedando por tanto el número de ítems en 22. En ningún caso se observaron correlaciones entre los ítems superiores a .90 .

Del análisis factorial exploratorio (AFE) realizado sobre la primera submuestra $(\mathrm{n} 1=$ 310) se obtuvieron dos factores que explicaban el 55.0\% de la varianza. Estos factores estaban formados por 7 ítems de los 22 iniciales, ya que éstos se fueron eliminando cuando su carga factorial no resultaba superior a .30 (Hair, Anderson, Tatham y Black, 2010). La medida de adecuación muestral de Kaiser-Meyer-Olkin ofreció un valor de .821 y la prueba de esfericidad de Bartlett resultó significativa $(2=772.713$; g.1. $=21 ; \mathrm{p}=.000)$. Los factores resultantes se denominaron "Uso del Tiempo" y "Disponibilidad de Tiempo". En la Tabla 1 se muestra la varianza explicada, el número de ítems que incluye cada factor y su saturación.

(c) Psy, Soc, \& Educ, 2019, Vol. 11(2) 
Tabla 1. Estructura factorial del cuestionario

\begin{tabular}{|c|c|c|}
\hline Factor & 1 & 2 \\
\hline $\mathrm{N}^{\mathrm{o}}$ Ítems Factor & 5 & 2 \\
\hline$\%$ Varianza Total Explicada Factor & $42.1 \%$ & $12.9 \%$ \\
\hline Ítems & \multicolumn{2}{|c|}{ Saturación } \\
\hline Nos demostramos cariño & .75 & \\
\hline Disfruto de los momentos que estamos juntos en pareja & .75 & \\
\hline Mi pareja se interesa por lo que hago en el día a día & .74 & \\
\hline Siento que puedo hablar con mi pareja de cualquier tema & .68 & \\
\hline Mi pareja se muestra disponible cuando la necesito & .67 & \\
\hline Disponemos del tiempo suficiente para poder comunicarnos & & .88 \\
\hline Pasamos suficiente tiempo juntos en pareja & & .68 \\
\hline
\end{tabular}

\section{Análisis factorial confirmatorio}

Los valores obtenidos con el análisis factorial confirmatorio sobre la segunda submuestra (n2 = 310) indicaron un ajuste óptimo del modelo, obteniéndose un valor de chi cuadrado significativo, $\chi 2=22.722$ (13), $\mathrm{p}<.045, \mathrm{CMIN} / \mathrm{DF}=1.748$ y los siguientes valores en los índices calculados: $\mathrm{GFI}=.98, \mathrm{RMSEA}=.049, \mathrm{SRMR}=.032, \mathrm{CFI}=.98, \mathrm{NFI}=.96, \mathrm{IFI}=.98$ y TLI $=$ .98. Los pesos de regresión estandarizados de los ítems oscilaron entre .58 y $.84(\mathrm{p} \leq .001)$, aportando evidencias de validez convergente para el modelo (Hair et al., 2006). El modelo quedó especificado tal como se presenta en la Figura 1. 


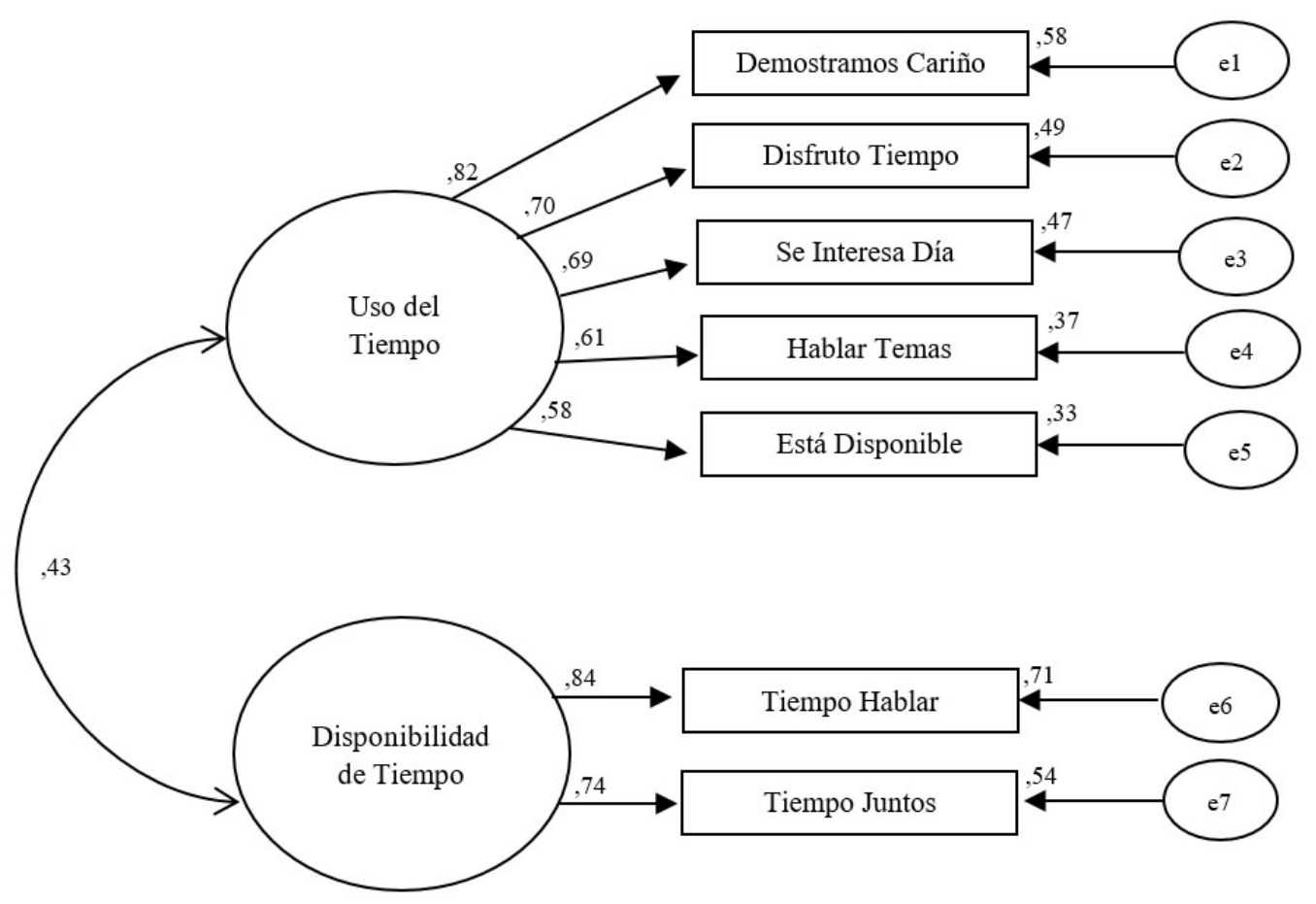

Figura 1. Análisis Factorial Confirmatorio (Submuestra 2)

\section{Consistencia interna o fiabilidad}

El coeficiente de fiabilidad Alfa de Cronbach del conjunto de los ítems fue de .80 y el de los factores resultantes fue de .82 (Factor 1) y .76 (Factor 2), lo cual indica una adecuada consistencia interna entre los elementos de ambos factores, ya que se considera que valores superiores a .70 o .80 son suficientes para garantizar la fiabilidad de la escala (Tornimbeni, Pérez y Olaz, 2008). Se comprobó, además, que dicho valor no aumentaba si se eliminaba alguno de los elementos.

\section{Validez concurrente}

El análisis de la validez concurrente calculada mediante la correlación de Pearson entre los factores resultantes y las variables externas seleccionadas como criterios, indicó que el ítem Estoy satisfecho con mi relación de pareja resultaba un buen predictor para el Factor $1 \mathrm{y}$ moderado para el Factor 2, ya que se obtuvieron unos valores en la correlación de Pearson de .70 y .33 respectivamente ( $\leq 0.00$ bilateral en ambos casos). El ítem Estoy satisfecho con la calidad del tiempo que compartimos mi pareja y yo, resultó un predictor moderado para ambos factores, obteniéndose unos valores en la correlación de Pearson de .52 y .48 respectivamente ( $\mathrm{p} \leq 0.00$ bilateral en ambos casos).

\section{Discusión}

Nuestra sociedad está inmersa, desde principios del siglo XX, en una serie de cambios demográficos que afectan en gran medida a la configuración de las familias (Escapa, 2017). A 
pesar de los cambios y evoluciones que ha experimentado, especialmente en las últimas décadas, la visión y la propia concepción social de la familia (por ejemplo con una mayor visibilización y aceptación de la diversidad) y, con ello, el ideal de relación de pareja, actualmente continúa siendo habitual identificar una relación de pareja con la presencia de fidelidad y exclusividad, tanto romántica como emocional, entre dos personas que han establecido un vínculo de apego basado en el cuidado mutuo, en el que ambas actúan como proveedores y dispensadoras de cuidados (emocionales, físicos, etc.) y que, si se desarrolla adecuadamente, supone una fuente de apoyo emocional y social que contribuye, en definitiva, al bienestar personal (Crowell y Treboux, 2001; Hazan y Diamond, 2000).

Como ya se contemplaba en la introducción de este trabajo, disponer de tiempo suficiente e invertirlo adecuadamente, supone un elemento clave a la hora de garantizar poder ofrecer a la otra persona la atención necesaria para ver cubiertas sus necesidades afectivas, de escucha o emocionales, así como parece condicionar de forma directa otras dimensiones tan fundamentales como la satisfacción con la propia relación o la comunicación que en ella se produce. En este sentido, tal y como recoge Sánchez Aragón (2009), en la medida en que las parejas son capaces de organizarse adecuadamente, tanto en lo emocional como en lo funcional, y que ambos puedan congeniar y compartir su tiempo con amigos y familiares, es más probable que se vea incrementada su estabilidad y seguridad en la relación.

En base a los beneficios que conlleva el correcto desarrollo de una relación de pareja, y la importancia de evaluar dimensiones como el tiempo disponible y empleado en ella para determinar el nivel de implicación y su relación con otros aspectos de la relación, se marcó el objetivo de diseñar un instrumento capaz de evaluar dicha dimensión. Tras los oportunos análisis factorial exploratorio y confirmatorio, se ha logrado obtener la Escala de Tiempo Compartido en la Pareja que, con únicamente siete ítems, repartidos en dos factores, mide de forma válida y fiable la dimensión de estudio planteada. Además, el análisis de confiabilidad y propiedades psicométricas generales parece indicar que puede ser de utilidad en contextos empíricos, lo que sumado a su brevedad y facilidad de aplicación, puede contribuir a catalogarla como una herramienta de interés para diversos ámbitos de investigación o intervención (educación, psicología, sociología, pedagogía social, etc.).

En cuanto a la estructura factorial de la escala, parece ser semejante a la de otras dimensiones de la relación de pareja, como la comunicación, al presentar más de un factor (Anderson y Sabatelli, 1992; Sánchez Aragón y Díaz-Loving, 2003), a diferencia de aquellas que, como la satisfacción con la relación, la literatura científica tiende a revalidar que mantienen una estructura unifactorial (Hendrick, 1988; Schumm, Nichols, Schectman y Grigsby, 1983; Urbano-Contreras, Iglesias-García y Martínez-González, 2017). Con respecto al número de ítems por factor, existe la tendencia a incluir al menos tres ítems por factor, pero ha quedado demostrado que esta práctica puede llegar a ser contraproducente al condicionar la estabilidad de los resultados (Velicer y Fava, 1998), sugiriendo la inclusión de un mínimo de dos ítem por factor, especialmente cuando los factores correlacionan o la escala cuenta con más de un factor (Raubenheimer, 2004).

Como aportaciones del estudio, se ha contado con una muestra que supera la tendencia en esta temática a realizar investigaciones con menos de 400 personas (Touliatos, Perlmutter y Holden, 2001). Además, se ha procurado que la población participante sea lo más diversa posible (zona geográfica, género, nivel de estudios, parejas con y sin hijos, etc.). Entre las 
limitaciones, no se han utilizado simultáneamente otros instrumentos ya validados ante la falta de escalas que midan exclusivamente esta dimensión de estudio y que hayan sido desarrolladas en el mismo contexto. Sin embargo, con el fin de suplir este déficit, se han seleccionado dos ítems que contribuyen a contrastar la validez de criterio o concurrente. Estas limitaciones, junto a otras posibles líneas de trabajo, plantean la posibilidad de continuar recogiendo muestra con el instrumento generado, incorporando otras zonas geográficas o mayor diversidad sociodemográfica (por ejemplo, con datos de parejas homosexuales o de edad más avanzada), así como complementar los resultados con la recogida de información cualitativa.

\section{Referencias}

Anderson, S. A. y Sabatelli, R. M. (1992). The differentiation on the family system scale (DIFS). The American Journal of Family Therapy, 20, 77-89.

Armenta-Huarte, C., Sánchez-Aragón, R. y Díaz-Loving, R. (2012). ¿De qué manera el contexto afecta la satisfacción con la pareja?. Suma Psicológica, 19(2), 51-62.

Capafóns, J. I. y Sosa, C. D. (2015). Relaciones de pareja y habilidades sociales: El respeto interpersonal. Psicologia Conductual, 23(1), 25-34.

Çetinkaya, S. K. y Gençdoğan, B. (2014). The relationship between marital quality, attitudes towards gender roles and life satisfaction among the married individuals. Psychology, Society, \& Education, 6(2), 94-112.

Cronbach, L. J. y Shavelson, R. J. (2004). My current thoughts on coefficient alpha and successor procedures. Educational and Psychological Measurement, 64(3), 391-418. doi: $10.1177 / 0013164404266386$

Crowell, J. A. y Treboux, D. (2001). Attachment and security in adult partnership. En C. Clulow (Ed.), Adult attachment and couple psychotherapy: The "secure base" in practice and research (pp. 28-42). Londres: Brunner-Routledge.

Curran, P. J., West, S. G. y Finch, J. F. (1996). The robustness of test statistics to nonnormality and specification error in confirmatory factor analysis. Psychological Methods, 1(1), 16-29.

Domínguez Folgueras, M. (2012). La división del trabajo doméstico en las parejas españolas. Un análisis de uso del tiempo. Revista internacional de sociología, 70(1), 153-179. doi: 10.3989/ris.2009.08.26

Escapa, S. (2017). Efectos del conflicto parental después del divorcio en el rendimiento educativo de los hijos. Revista Española de Investigaciones Sociológicas, (158), 41-58. doi: $10.5477 / \mathrm{cis} / \mathrm{reis} .158 .41$

Fabrigar, L., MacCallum, R., Wegener, D. y Strahan, E. (1999). Evaluating the use of exploratory factor analysis in psychological research. Psychological Methods, 4(3), 272-299. doi: $/ 10.1037 / / 1082989 x .4 .3 .272$

Faus-Bertomeu, A. y Gómez-Redondo, R. (2017). Determinantes socioculturales del deseo sexual femenino. Revista Española de Investigaciones Sociológicas, (160), 61-78. doi: $10.5477 / \mathrm{cis} /$ reis. 160.61

Finch, H. (2006). Comparison of the Performance of Varimax and Promax Rotations: Factor Structure Recovery for Dichotomous Ítems. Journal of Educational Measurement, 43, 39-52. doi: 10.1111/j.1745-3984.2006.00003.x

Fuertes Martín, J. A. (2000). Relaciones afectivas y satisfacción sexual en la pareja. Revista de Psicología Social, 15(3), 343-356.

García Román, J. (2013). El tiempo con la familia en las parejas de doble ingreso. Un análisis a partir de la Encuesta de Empleo del Tiempo 2009-2010. Estadística Española, 55(182), 259-282. 
Gil Pascual, J. A. (2011). Técnicas e instrumentos para la recogida de información. Madrid: UNED.

Hair, J. F., Anderson, R. E., Tatham, R. L. y Black, W. C. (2010). Análisis multivariante. Madrid: Pearson Prentice Hall.

Hair, J. F., Black, W. C., Babin, B. J., Anderson, R. E. y Tatham, R. L. (2006). Multivariate data analysis (7th ed.). Upper Saddle River, NJ: Pearson Prentice Hall.

Hazan, C. y Diamond, L. M. (2000). The place of attachment in human mating. Review of General Psychology, 4(2), 186-204.

Hendrick, S. S. (1988). A generic measure of relationship satisfaction. Journal of Marriage and Family, 50, 93-98. doi: 10.2307/352430

Hidalgo, M. V. y Menéndez, S. (2003). La pareja ante la llegada de los hijos e hijas. Evolución de la relación conyugal durante el proceso de convertirse en padre y madre. Infancia y Aprendizaje, 26(4), 469-483. doi: 10.1174/021037003322553851

Hidalgo, M. V. y Menéndez, S. (2009). Apoyo a las familias durante el proceso de transición a la maternidad y la paternidad. Familia: Revista de Ciencias y Orientación Familiar, 38, 133-152.

Hoelter, J. W. (1983). Factorial invariance and self-esteem: Reassessing race and sex differences. Social Forces, 61(3), 834-846. doi: 10.1093/sf/61.3.834

Ledermann, T., Bodenmann, G., Rudaz, M. y Bradbury, N. B. (2010). Stress, communication, and marital quality in couples. Family Relations, 59(2), 195-206. doi: 10.1111/j.17413729.2010.00595.x

Leggett, D. G., Roberts-Pittman, B., Byczek, S. y Morse, D. T. (2012). Cooperation, conflict and marital satisfaction: Bridging theory, research, and practice. The Journal of Individual Psydiology, 68(2), 182-199.

Little, R. J. A. (1988). A test of missing completely at random for multivariate data with missing values. Journal of the American Statistical Association, 83(404), 1198-1202.

Markland, D. (2007). The golden rule is that there are no golden rules: A commentary on Paul Barrett's recommendations for reporting model fit in structural equation modelling. Personality and Individual Differences, 42(5), 851-858. doi: 10.1016/j.paid.2006.09.023

Martínez-Álvarez, J. L., Fuertes-Martín, A., Orgaz-Baz, B., Vicario-Molina, I. y González-Ortega, E. (2014). Vínculos afectivos en la infancia y calidad en las relaciones de pareja de jóvenes adultos: el efecto mediador del apego actual. Anales de Psicología, 30(1), 211-220. doi: 10.6018/analesps.30.1.135051

Mora Torres, M., Gómez Cortés, M. y Rivera Heredia, M. (2013). La satisfacción marital y los recursos psicológicos en las parejas con y sin hijos pequeños en pro del bienestar familiar. Uaricha, 10(22), 79-96.

Moreno-Colom, S., Ajenjo Cosp, M. y Borràs Català, V. (2018). La masculinización del tiempo dedicado al trabajo doméstico rutinario. Revista Española de Investigaciones Sociológicas, (163), 41-58. doi: 10.5477/cis/reis. 163.41

Padilla González, L., Armas Vargas, E., García Pérez, L. y Aguiar González, D. (2012). Percepción de la comunicación en la pareja en víctimas de violencia de género (CPC-RC-PAREJA). En R. Quevedo-Blasco y V. J. Quevedo Blasco (Comp.), Avances en Psicología Clínica (pp. 766770). Santander: Asociación Española de Psicología Conductual.

Raubenheimer, J. (2004). An item selection procedure to maximize scale reliability and validity. Journal of Industrial Psychology, 30(4), 59-64.

Ríos González, J. A. (2005). Los ciclos vitales de la familia y la pareja: ¿crisis u oportunidades? Madrid: CCS.

Sánchez Aragón, R. (2009). Expectativas, percepción de estabilidad y estrategias de mantenimiento en las relaciones amorosas. Enseñanza e investigación en psicología 14(2), 229-243. 
Sánchez Aragón, R. y Díaz-Loving, R. (2003). Patrones y estilos de comunicación de la pareja: Diseño de un inventario. Anales de Psicología, 19(2), 257-277.

Schumm, W. A., Nichols, C. W., Schectman, K. L. y Grigsby, C. C. (1983). Characteristics of responses to the Kansas Marital Satisfaction Scale by a sample of 84 married mothers. Psychological Reports, 53(2), 567-572. doi: 10.2466/pr0.1983.53.2.567

Tabachnick, B. G. y Fidell, L. S. (2001). Using multivariate statistics. Northridge: Pearson.

Torres, A. C. (2004). Vida Conjugal e Trabalho. Oeiras, Portugal: Celta Editora.

Tornimbeni, S., Pérez, E. y Olaz, F. (2008). Introducción a la psicometría. Buenos Aires: Paidós.

Touliatos, J., Perlmutter, B. F. y Holden, G. W. (2001). Handbook of family measurement techniques. USA: Sage.

Urbano Contreras, A. (2018). Relaciones de pareja y sus implicaciones familiares: Análisis de necesidades socioeducativas. Tesis doctoral. Universidad de Oviedo.

Urbano-Contreras, A., Iglesias-García, M. T. y Martínez-González, R. A. (2017). Development and validation of the Satisfaction in couple relationship scale (SCR). Contemporary Family Therapy, 39(1), 54-61. doi: 10.1007/s10591-016-9400-z

Urbano-Contreras, A., Iglesias-García, M. T. y Martínez-González, R. A. (2019). Satisfacción general y sexual con la relación de pareja en función del género. Revista Española de Investigaciones Sociológicas, (165), 143-158. doi: 10.5477/cis/reis.165.143

Urbano-Contreras, A., Martínez-González, R. A. e Iglesias-García, M. T. (2018). Parenthood as a Determining Factor of Satisfaction in Couple Relationships. Journal of Child and Family Studies. doi: 10.1007/s10826-017-0990-3

Van Ginkel, J. R. y Van der Ark, L. A. (2005). SPSS syntax for missing value imputation in test and questionnaire data. Applied Psychological Measurement, 29(2), 152-153.

Velicer, W. F. y Fava, J. L. (1998). Effects of variable and subject sampling on factor pattern recovery. Psychological Methods, 3(2), 231-251. doi: 10.1037/1082-989X.3.2.231

Wals, F., Romera, E. M. y Viejo, C. (2015). Influencia de la auto-eficacia social y el apoyo social en la calidad de las relaciones de pareja adolescentes. Psychology, Society, \& Education, 7(1), 71-84. 


\section{Anexo 1. Versión definitiva de la Escala de Tiempo Compartido en la Pareja (TCP)}

La finalidad de este cuestionario es recoger información sobre el tiempo compartido en pareja. Es anónimo y se garantiza la confidencialidad de la información. No se trabajará con respuestas individuales, sino con el conjunto obtenido tras aplicarlo a muchas personas. Para responder al cuestionario tenga en cuenta su relación de pareja actual. Por favor, lea con atención cada pregunta y señale con una $\mathrm{X}$ su respuesta.

1. Sexo: a) Mujer $\square \quad$ b) Hombre $\square$

2. Edad:

3. País de nacimiento: 4. Municipio actual de residencia:

5. Nivel de estudios: a) Sin estudios

b) EGB/E.S.O.

c) Bachillerato/FP

d) Universitarios

6. Situación laboral:
a) Desempleado
c) Estudiante $\square$
e) Trabajo en la Administración Pública
b) Autónomo $\square$
d) Trabajador en empresa privada
f) Otra (indique cuál):

7. Tipo de pareja: a) Hombre-mujer

b) Hombre-hombre $\square$

c) Mujer-mujer $\square$

8. Estado civil con respecto a su pareja actual: a) Soltero

b) Pareja de hecho

c) Casado $\square$

9. ¿Cuánto tiempo de relación lleva con su pareja? (indicar años y meses):

10. Convive con (indicar todos los miembros de la unidad familiar):

11. En caso de tener hijos:

\begin{tabular}{|l|l|l|l|l|}
\hline & Número & Edad de los hijos & Sexo & ¿Cuántos viven en casa? \\
\hline En común con mi pareja & & & & \\
\hline Míos con otras parejas & & & & \\
\hline De mi pareja con otras parejas & & & & \\
\hline
\end{tabular}

12. Si convive actualmente con su pareja, ¿Cuánto tiempo llevan conviviendo? (indicar años y meses):

13. A parte de su relación actual, ¿Ha tenido otras relaciones de pareja? (indicar número):

Por favor, valore marcando con una X su opinión sobre las siguientes afirmaciones:

1) Totalmente en desacuerdo (Nunca)

3) Acuerdo (Casi siempre)

2) Desacuerdo (A veces)

4) Totalmente de acuerdo (Siempre)

\begin{tabular}{|c|c|c|c|c|}
\hline Tiempo Compartido en la Pareja (TCP) & 1 & 2 & 3 & 4 \\
\hline 1. Nos demostramos cariño & & & & \\
\hline 2. Disfruto de los momentos que estamos juntos en pareja & & & & \\
\hline 3. Mi pareja se interesa por lo que hago en el día a día & & & & \\
\hline 4. Siento que puedo hablar con mi pareja de cualquier tema & & & & \\
\hline 5. Mi pareja se muestra disponible cuando la necesito & & & & \\
\hline 6. Disponemos del tiempo suficiente para poder comunicarnos & & & & \\
\hline 7. Pasamos suficiente tiempo juntos en pareja & & & & \\
\hline
\end{tabular}


Escala de Tiempo Compartido en la Pareja (TCP)

(C) Psy, Soc, \& Educ, 2019, Vol. 11(2) 